AIAA-2002-3273

\title{
Virtual Shaping of a Two-dimensional NACA 0015 Airfoil Using Synthetic Jet Actuator
}

\author{
Fang-Jenq Chen" and George B. Beeler" \\ NASA Langley Research Center \\ Hampton, VA 23681-0001
}

\begin{abstract}
The Aircraft Morphing Program at NASA Langley envisions an aircraft without conventional control surfaces. Instead of moving control surfaces, the vehicle control systems may be implemented with a combination of propulsive forces, micro surface effectors, and fluidic devices dynamically operated by an intelligent flight control system to provide aircraft maneuverability over each mission segment. As a part of this program, a two-dimensional NACA 0015 airfoil model was designed to test mild maneuvering capability of synthetic jets in a subsonic wind tunnel. The objective of the experiments is to assess the applicability of using unsteady suction and blowing to alter the aerodynamic shape of an airfoil with a purpose to enhance lift and/or to reduce drag. Synthetic jet actuation at different chordwise locations, different forcing frequencies and amplitudes, under different freestream velocities are investigated. The effect of virtual shape change is indicated by a localized increase of surface pressure in the neighborhood of synthetic jet actuation. That causes a negative lift to the airfoil with an upper surface actuation. When actuation is applied near the airfoil leading edge, it appears that the stagnation line is shifted inducing an effect similar to that caused by a small angle of attack to produce an overall lift change.
\end{abstract}

\section{NOMENCLATURE}

$C_{p} \quad$ pressure coefficient

$C_{d p} \quad$ pressure drag coefficient

$C_{l, u s} \quad$ upper surface lift coefficient

$C_{\mu} \quad$ jet momentum coefficient $=2(h / c)^{* *}\left(v_{r m s} / U_{\infty}\right)^{2}$

${ }^{\overline{ }}$ Senior Aerospace Engineer.

Copyright 2002 by the American Institute of Aeronautics and Astronautics, Inc. No Copyright is asserted in the United States under Title 17, U.S. Code. The U.S. Government has a royalty-free license to exercise all rights under the copyright claimed herein for Governmental purposes. All other rights are reserved by the copyright owner.

\author{
$\Delta C_{l} \quad$ change of lift coefficient due to actuation \\ c airfoil chord \\ $E_{\text {eff }} \quad$ effective voltage \\ $E_{\text {in }} \quad$ amplitude of sinusoidal input voltage \\ $f \quad$ frequency \\ $h \quad$ jet exit slot width \\ $I_{\text {eff }} \quad$ effective current \\ $P \quad$ active power, Eq. (1) \\ $Q \quad$ reactive power, Eq. (2) \\ $R e_{c} \quad$ Reynolds number based on chord length \\ $U$ mean streamwise velocity \\ $u$ broadband rms streamwise fluctuating velocity \\ $v \quad$ phased-averaged jet velocity \\ $x \quad$ streamwise or chordwise coordinate \\ $z \quad$ spanwise coordinate \\ $\theta$ phase angle of voltage with respect to current
}

\section{$\underline{\text { Subscripts }}$}

max maximum value during actuator blowing cycle

mean mean value

min minimum value during actuator suction cycle

$r m s$ root-mean-square value

$\infty \quad$ freestream

\section{INTRODUCTION}

New technologies and advanced materials, both nearly in hand and in early development, offer the potential to create revolutionary advances in aerospace vehicles with significantly greater performance and maneuvering compared to conventional approaches.

Conventional airfoils of aerospace vehicles have been designed for a single flight condition and then modified to cover multiple flight conditions. This is done through the use of control surfaces, such as ailerons and flaps, spoilers, and variable wing sweep. Variable wing sweep affects changes in the local flow field by altering the flow velocity normal to the leading 
edge of the airfoil. The control surfaces and spoilers affect changes in the flow field by directly varying the camber on certain regions of the airfoil, thereby causing changes in the baseline aerodynamic characteristics of the entire airfoil.

The Aircraft Morphing Program at NASA Langley has been initiated with the goal to develop next generation aerospace vehicles by mimicking Nature. Efforts include providing muscle-like actuators to change aerodynamic form on demand, nervous-systemlike sensing, self-healing materials, and adaptive faulttolerant controls to enhance flight safety. One approach envisions future vehicles without conventional control surfaces that could lead to significant weight reductions or decrease the aircraft signature. Instead of moving control surfaces, the aerodynamic control systems may be implemented with a combination of thrust vectoring propulsion, adaptive micro-machined surface effectors, and distributed fluidic devices dynamically operated by an intelligent control system to provide aircraft maneuverability under different flight conditions. As a part of this program, a two-dimensional NACA 0015 airfoil model was designed to test mild maneuvering capability of synthetic jets in the NASA Langley $2 \mathrm{ft} \times$ $3 \mathrm{ft}$ Tunnel. The objective of the experiments is to assess the applicability of using unsteady suction and blowing of synthetic jets to alter the aerodynamic shape of an airfoil to enhance lift and/or to reduce drag.

Previous preliminary tests with a piston-type actuator indicate that the actuator has a net effect on the boundary-layer flow of producing a local net increase in the displacement thickness of the flow. Hence an effective shape change could approximate the effect of the synthetic jet actuator. Most recent works ${ }^{1-6}$ on airfoil aerodynamic manipulation using synthetic jets has focused on the control of flow separation at moderate and large angles of attack. The experimental work done by Chatlynne et $\mathrm{al}^{7}$ showed that it is possible to modify the apparent aerodynamic shape of an airfoil at low angles of attack when the baseline flow is fully attached. However, this virtual aero-shaping was achieved by combining the activation of a highfrequency synthetic jet actuator placed downstream from a miniature surface-mounted passive obstruction (a little fence). This combination introduces a small stationary re-circulating flow region next to the surface, which displaces the local streamlines sufficiently to modify the local pressure distribution. The modified flow results in a significant reduction in pressure drag with a minimal loss of lift. Physically, the function of the synthetic jet actuator in this application is to force the separated flow downstream of the little fence to reattach to the surface.
The work described in the present paper focuses on the aerodynamic virtual shaping of an airfoil at zero angle of attack, using only synthetic jets without additional fixtures. This represents an examination of the fluidic modification of aero-surfaces that does not necessarily rely on coupling to flow instability such as that inherently in separated flows. The effects of virtual shaping are investigated with synthetic jet actuation at different chordwise locations, different forcing frequencies and amplitudes, under different freestream velocities. Characteristics of electric power consumption of the synthetic jet actuator are also reported in this paper.

\section{APPARATUS}

\subsection{Wind Tunnel Facility}

The experiment was conducted in the $2 \mathrm{ft} \times 3 \mathrm{ft}$ Low-Speed Wind Tunnel located at the NASA Langley Research Center. The tunnel is a closed-loop type with a $10: 1$ contraction ratio. The test section is $91.4 \mathrm{~cm}$ wide by $61.0 \mathrm{~cm}$ high by $6.1 \mathrm{~m}$ long. The turbulencereduction devices upstream of the contraction consist of a honeycomb followed by four stainless-steel screens. A vane-axial fan powered by a 30 horsepower DC motor is used to drive the tunnel. Speeds of approximately $45 \mathrm{~m} / \mathrm{sec}$ are attainable in the test section with measured turbulence intensities, $u / U_{\infty}$, of approximately $0.1 \%$ in the range of $0.1<f<400 \mathrm{~Hz}$. The test-section ceiling and floor are adjustable to achieve a desired streamwise pressure gradient and accommodate various test conditions. Further details of the facility have been previously published. ${ }^{8}$

\subsection{Airfoil Model}

The two-dimensional NACA 0015 airfoil model has the dimensions of $91.4 \mathrm{~cm}$ span and $91.4 \mathrm{~cm}$ chord. The model was made with a $0.3 \mathrm{~cm}$ thick lay-up of fiberglass skin supported by four internal chordwise ribs machined from 6061-T6 aluminum alloy. There are six chordwise locations for installation of a synthetic jet actuator with a long exhaust slot centered spanwise on the model. Figures 1(a), (b) and (c) show a side view, a cut-away view and a photograph of the airfoil model. The model was mounted to external mounts through the tunnel sidewalls by a spanwise-extended steel tube, as shown in the center of Figure 1(c). The surface finish on the airfoil surface was 1 micron or better.

Three rows of streamwise pressure taps are located on the midspan, the right- and the left-quarter-spans on both upper and lower surfaces. The pressure taps are staggered in each row to minimize streamwise interference. The nominal orifice diameter of the pressure taps is $0.05 \mathrm{~cm}$. Three 32-port 10 -inch water- 
column ESP modules were connected to pressure taps to get the surface static pressure readings. Ten dynamic pressure transducers, two Kulite XCS-062-5D and eight Endevco $8510 \mathrm{~B}-1$, were distributed along the midspan with nine on the upper surface and one on the lower surface. Locations of the pressure taps and the dynamic pressure transducers are shown in the sketches of Figures 2(a) and (b). One row of spanwise pressure taps distributed near the trailing edge approximately at $x / c=$ 0.95, also shown in Figure 2(a), was used to check possible tunnel-sidewall contamination of the surface pressure readings.

\subsection{Synthetic Jet Actuator}

A long two-dimensional synthetic jet actuator, as shown in Figure 3, was installed across the airfoil span at one of six different chordwise locations for the test. These six actuator locations are at $x / c=-0.015,0.1$, $0.15,0.3,0.5$, and 0.75 (the negative value was on the bottom side of the airfoil). One of the test purposes was to determine the optimal placement of the actuator on the airfoil for virtual shape change. The actuator consists of a continuous cavity enclosed by 14 pairs of piezoelectric diaphragms. Each pair of piezoelectric diaphragms was operated with a $180^{\circ}$ phase differential at the same sinusoidal voltage and frequency. With actuation, a synthetic jet issued from a two-dimensional slot that is $76.2 \mathrm{~cm}$ long by $0.5 \mathrm{~mm}$ wide on the top plate of the device. Six top plates with different curvatures were made to fair the actuator to different chordwise locations on the airfoil model. A multichannel attenuation unit was used to obtain the best uniformity of the synthetic jet across the airfoil span by adjusting the amplitude of power input to each pair of piezoelectric diaphragms.

\section{CHARACTERISTICS OF ACTUATOR}

The strength of synthetic jets generally is not uniform along the $76.2 \mathrm{~cm}$ spanwise slot due to irregularity in the piezoelectric diaphragms. After careful tuning with the attenuation unit, the best uniformity of the synthetic jet at the maximum performance condition (defined later) was obtained and shown in Figure 4. It shows slight variations of jet velocities along the slot centerline on the jet exit surface in the quiescent environment. The jet is slightly stronger in the position at top of the center of each piezoelectric diaphragm pair than that at top of the interval between two diaphragm pairs.

Typical variations of jet velocities at the maximum performance condition with respect to the forcing frequency, in the range of $200 \mathrm{~Hz}$ to $1700 \mathrm{~Hz}$, at the slot center on jet exit surface, are shown in Figure 5. It presents two distinguished peaks at the frequencies around $600 \mathrm{~Hz}$ and $1300 \mathrm{~Hz}$. These two peak frequencies correspond to the natural (mechanical) frequency of the piezoelectric diaphragm and the Helmholtz (acoustic) frequency of the actuator cavity, respectively. More detailed discussions of these resonant frequencies were given in a previous paper. ${ }^{9}$

The characteristics of electric power used to drive the piezoelectric diaphragms of the actuator at the forcing frequencies of 600 and $1300 \mathrm{~Hz}$ are shown in Figures 6 and 7, respectively. The electric power level was adjusted by varying the amplitude of sinusoidal voltage input through the multi-channel attenuation unit to a high-voltage power amplifier with a nominal gain of 100 before sending to the actuator. The input voltage, $E_{\text {in }}$, is the peak-to-peak amplitude of the sinusoidal voltage generated by a universal function generator. Figures 6(a) and 7(a) show variations of the effective values (in terms of standard deviations) of current, $I_{e f f}$, voltage, $E_{e f f}$, and the phase angle, $\theta$, of voltage with respect to current, with the input voltage, $E_{i n}$. Generally the effective current and voltage increase with the input voltage but the phase angle decreases slightly from $+90^{\circ}$. It indicates that the piezoelectric diaphragm deviates from a perfect capacitance device as the input voltage increases. Figures $6(\mathrm{~b})$ and 7(b) show relations between the jet energy, in terms of variance of jet velocity, $\left(v_{m s}\right)^{2}$, the active $(P)$ and the reactive $(Q)$ electric powers with the input voltage, $E_{\text {in }}$. Note that the reactive power is plotted as $Q / 4$ in the figures in order to compile data in the same scale. The active power, $P$, and the reactive power, $Q$, are defined as $^{10}$

$$
\begin{aligned}
& P=E_{\text {eff }} I_{e f f} \cos \theta \\
& Q=E_{\text {eff }} I_{\text {eff }} \sin \theta
\end{aligned}
$$

The performance of the piezoelectric diaphragm (hence, the actuator) can be enhanced by an offset of the input voltage that is shown as a step jump for the input voltage equal to and greater than 2.0 volts in the figures. The maximum performance condition of the piezoelectric diaphragm and the actuator was achieved at the input voltage of 2.3 volts with an offset of 0.6 volts. Data at the maximum performance condition are plotted in Figures 8(a) and 8(b) against the forcing frequency. The active power is closely correlated with the jet output as seen in the (b) plot of Figures 6,7 and 8 . The reactive power is due to the capacitive nature of the actuator. The magnitude of the reactive power is always greater than the active power in this device. It is very interesting to see that the performance peaks, approximately at 600 and $1300 \mathrm{~Hz}$, happen when the

American Institute of Aeronautics and Astronautics 
phase angle between voltage and current is small and the reactive power is low, as shown in Figures 8(a) and 8 (b). The active power is very indicative of the phase matching of the driving frequency to the resonance mode of the synthetic jet as seen by the peaks in active power and jet output in Figure $8(\mathrm{~b})$. The differing character of the peak active power at the Helmholtz resonance versus the mechanical resonance may be indicative of the more complex non-linear coupling in the system. The data hints at the potential of monitoring the active power as a potential tuning tool to maintain the excitation near resonance of the actuator.

\section{VIRTUAL SHAPING USING SYNTHETIC JETS}

The effect of virtual shape change on the airfoil model was detected by comparing the measurements of airfoil surface pressures with the synthetic jet actuation on and off. Typical variations of the surface pressure coefficient, $C_{p}$, with actuation on and off on the upper and the lower surfaces are shown in Figures 9(a) and 9 (b), respectively. Customarily the $C_{p}$ scale is inverted in the plot. It clearly indicates a localized increase of the surface pressure in the neighborhood of synthetic jet actuation. That causes a negative lift on the airfoil with the actuator located on the upper surface. This negative lift change is consistent with numerical simulations of Hassan. ${ }^{11}$ Note that, at zero angle of attack, there is zero lift for the baseline (without actuation) NACA 0015 airfoil due to the geometric symmetry. The computed $C_{p}$ from the Reynolds Averaged Navier-Stokes solver CFL3D ${ }^{12}$ for the baseline airfoil (also included in the figures) show a good match with the measured nocontrol data. There is no discernible spanwise variation of $C_{p}$ by comparing the three rows of streamwise pressure readings along the midspan, the right- and the left-quarter-spans on both upper and lower surfaces, denoted as UpperC, UpperR, UpperL, LowerC, LowerR, and LowerL in the figures. Typical $C_{p}$ distributions from spanwise pressure taps near the trailing edge with the synthetic jet actuation on/off, under the same flow conditions as Figure 9, are presented in Figure 10. It indicates no discernible sidewall contamination on the $C_{p}$ data.

Presumably virtual shaping is by nature an inviscid local phenomena rather than a convective one such as separation control. The status of the boundary layer, either laminar or turbulent, is assumed to be a minor effect on virtual shaping. Power spectra of pressure fluctuations obtained by dynamic pressure transducers, such as the one shown in Figure 11, were used to verify the boundary layer conditions. To investigate the effect of boundary-layer transition on virtual shaping, a 1.27 cm wide tape with randomly distributed $\# 35$ Grit was used to trip the boundary layer at $x / c=0.12$ on the airfoil upper surface. Figure 12 shows that the boundary-layer trip moved the transition boundary (laminar data on left-hand side) to a lower freestream velocity but no discernible difference on airfoil lift change due to synthetic jet actuation for data between trip (turbulent data) and no-trip (laminar data) transition boundaries except normal data scattering.

Typical variations of airfoil lift change with forcing frequency, when the actuator was driven at the maximum performance condition (i.e., input 2.3 volts and offset 0.6 volts), are shown in Figure 13. The change of lift coefficient, $\Delta C_{l}$, is presented as a percentage of the airfoil upper surface lift, $C_{i, u s}$, that has a nominal value of 0.23 . Two negative lift peaks in Figure 13 correspond to the jet energy peaks as shown in Figure 8(b). Variations of airfoil lift change, $\Delta C_{l} / C_{l, u s}$, with jet momentum coefficient, $C_{\mu}$, are presented in Figures 14(a) to 14(d) for the actuator located at $x / c=0.1,0.15,0.3$ and 0.5 on the airfoil upper surface. Data are presented for the actuator operated at peak forcing frequencies of $600 \mathrm{~Hz}$ and $1300 \mathrm{~Hz}$ with varying input voltages and freestream velocities. Generally the lift change increases with $C_{\mu}$ and actuation chordwise coordinate after $x / c=0.15$. However, the lift change decreases for actuation at $x / c=$ 0.75 as shown in Figure 15. Numerical computations verified that the laminar boundary layer separation occurred at $x / c=0.53$. It indicates that virtual shaping by synthetic jet actuation is much less effective in separated flow. In the range of data tested, the maximum lift change was -0.015 that resulted in a $-6 \%$ change in $\Delta C_{l} / C_{l, u s}$ with actuation at $x / c=0.5$, as shown in Figure 14(d). An attempt to correlate the data shown in Figures 14(a) to 14(d) is presented in Figure 16 with a fitted line of $Y=-17^{*} X^{0.25}$ where $X$ and $Y$ represent the lumped variables for the $x$ and $y$ coordinates, respectively. The correlation result implies that virtual shaping by synthetic jet actuation is inversely proportional to the Reynolds number and the local logarithmic pressure gradient but increases (negative lift) with the jet momentum coefficient.

When synthetic jet actuation was near the leading edge $(x / c=-0.015)$, it appeared that the stagnation line was shifted inducing an effect similar to that caused by a small positive angle of attack to produce an overall positive lift increase. Figures 17(a) and 17(b) show typical $C_{p}$ distributions on the airfoil upper and lower surfaces, respectively. Variations of lift increase with respect to the jet energy and the forcing frequency is compiled in Figure 18 with the actuator driven at the maximum performance condition. The increase of lift does not closely follow the peak of jet energy because

American Institute of Aeronautics and Astronautics 
the increase of lift is saturated at $C_{\mu} \approx 0.003$, when $U_{\infty} \approx$ $10 \mathrm{~m} / \mathrm{sec}$, as indicated in Figure 19. Variations of lift increase with jet momentum coefficient under different freestream velocities are shown in Figures 20(a) to 20 (d) for data at forcing frequencies of $600 \mathrm{~Hz}$ and $1300 \mathrm{~Hz}$ only. Lift increase and jet momentum coefficient are correlated as a function of Reynolds number and presented in Figure 21 with two fitted lines, $Y=230+72^{*} \log X$ and $Y=260+72^{*} \log X$, for forcing frequencies of $600 \mathrm{~Hz}$ and $1300 \mathrm{~Hz}$, respectively. Where $X$ and $Y$ represent the lumped variables for the $x$ and $y$ coordinates.

The effect of virtual shaping on the airfoil pressure drag coefficient, $C_{d p}$, is never greater than 0.002 under any circumstance of this investigation. Most of the time it is negligible therefore not included for discussion.

\section{SUMMARY}

Aerodynamic virtual shaping of a two-dimensional NACA 0015 airfoil were investigated in the NASA Langley $2 \mathrm{ft} \times 3 \mathrm{ft}$ Tunnel, using a synthetic jet actuator at different chordwise locations, different forcing frequencies and amplitudes, under different freestream velocities. Important characteristics of electric power consumption of the synthetic jet actuator were also reported. Results are summarized as follows:

1. Synthetic jet energy is closely correlated to the active power consumed by the actuator. The data hints at the potential of monitoring the active power as a potential tuning tool to maintain the excitation near resonance of the actuator.

2. Performance of the synthetic jet actuator increases when the magnitude of reactive power and the phase lag of voltage to current are decreasing.

3. Synthetic jet actuation on the airfoil upper surface causes a localized increase of surface pressure that results in a negative lift to the airfoil. In the range of data tested, the maximum lift change was -0.015 that resulted in a $-6 \%$ change in $\Delta C_{l} / C_{l, u s}$ with actuation at $50 \%$ of chord.

4. Effect of virtual shaping is drastically decreased when synthetic jet actuation is applied under separated flow.

5. The stagnation line is shifted by synthetic jet actuation near the airfoil leading edge, inducing an effect similar to that caused by a small angle of attack to produce an overall lift change.

Synthetic jets represent a breakthrough in actuator technology, but further development is required to substantially increase jet momentum output before applying them to real flight vehicles.

\section{ACKNOWLEDGEMENT}

The authors would like to acknowledge the assistance of Dr. Ponnampalam Balakumar of the NASA Langley Research Center to provide numerical computational results used in this paper.

\section{REFERENCES}

1. Amitay, M., Smith, B. L., and Glezer, A., "Aerodynamic Flow Control Using Synthetic Jet Technology", AIAA Paper 98-0208, 1998.

2. Smith, D. R., Amitay, M., Kibens, V., Parekh, D., and Glezer, A., "Modification of Lifting Body Aerodynamics Using Synthetic Jet Actuators", AIAA Paper 98-0209, 1998.

3. Seifert, A., Eliahu, S., Greenblatt, D., and Wygnanski, I., "Use of Piezoelectric Actuators for Airfoil Separation Control", AIAA Journal, Vol. 36, No. 8, pp. 1535-1537, 1998.

4. Crook, A., Sadri, A. M., and Wood, N. J., "The Development and Implementation of Synthetic Jets for the Control of Separated Flow", AIAA Paper 99-3176, 1999.

5. Amitay, M., Smith, D. R., Kibens, V., Parekh, D., and Glezer, A., "Aerodynamic Flow Control over an Unconventional Airfoil Using Synthetic Jet Actuators", AIAA Journal, Vol. 39, No. 3, pp. 361370, 2001.

6. Honohan, A. M., Amitay, M., and Glezer, A., "Aerodynamic Control Using Synthetic Jets", AIAA Paper 2000-2401, 2000.

7. Chatlynne, E., Rumigny, N., Amitay, M., and Glezer, A., "Virtual Aero-Shaping of a Clark-Y Airfoil Using Synthetic Jet Actuators", AIAA Paper 2000-0732, 2000.

8. King, R. A., "Receptivity and Growth of Two- and Three-Dimensional Disturbances in a Blasius Boundary Layer", Ph.D. thesis, Massachusetts Institute of technology, Cambridge, MA, 2000.

9. Chen, F.-J., Yao, C., Beeler, G. B., Bryant, R. G., and Fox, R. L., "Development of Synthetic Jet Actuators for Active Flow Control at NASA Langley", AIAA Paper 2000-2405, 2000.

10. Fitzgerald, E., Higginbotham, D. E., and Grabel, A., "Basic Electrical Engineering", Fifth Edition, McGraw-Hill Book Company, 1981.

11. Hassan, A. A., "Numerical Simulations and Potential Applications of Zero-Mass Jets for Enhanced Rotorcraft Aerodynamic performance", AIAA Paper 98-0211, 1998.

12. Rumsey, C., Biedron, R., and Thomas, J., "CFL3D: Its History and Some Recent Applications", NASA TM-112861, May 1997. 


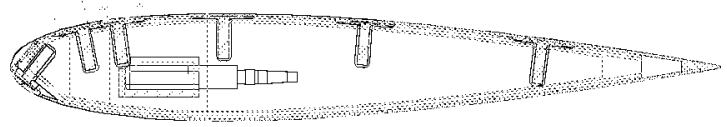

(a) Side view of airfoil model.

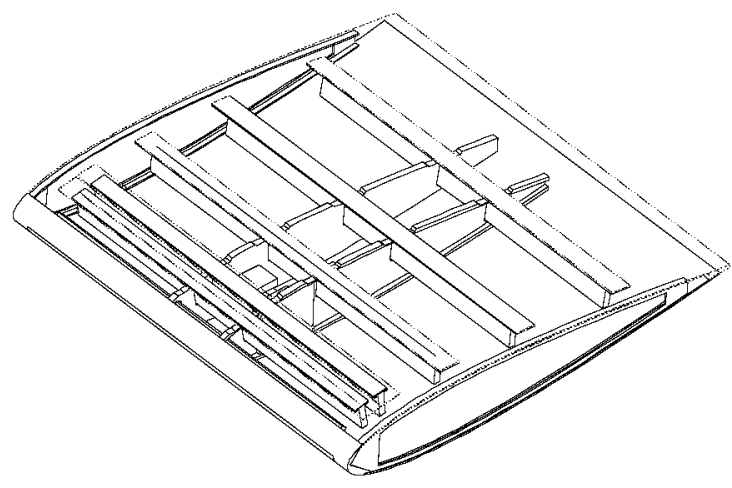

(b) Cut-away view of airfoil model.

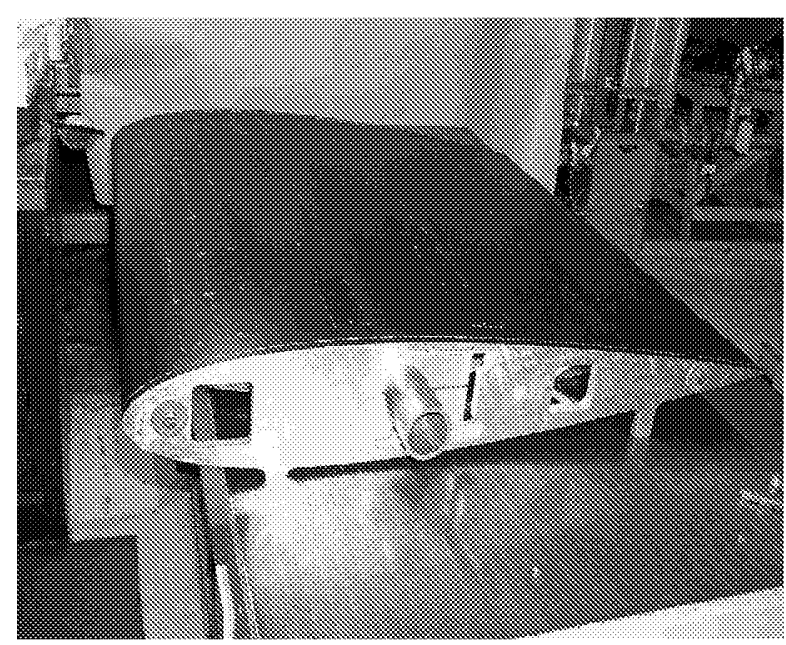

(c) Photograph of airfoil model on bench top.

Figure 1. NACA 0015 two-dimensional airfoil model for virtual shaping test.

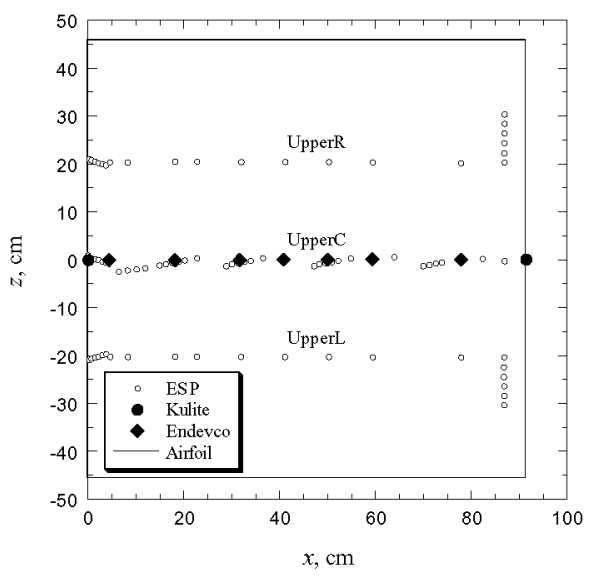

(a) Airfoil upper surface.

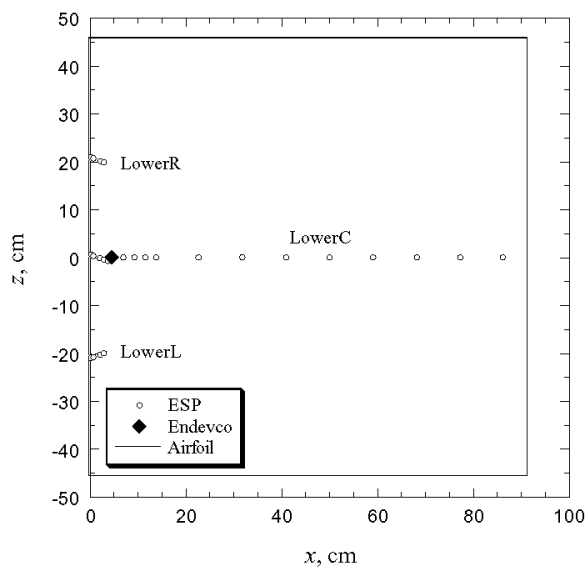

(b) Airfoil lower surface.

Figure 2. Distribution of ESP pressure ports and dynamic pressure transducers on the airfoil model surface.

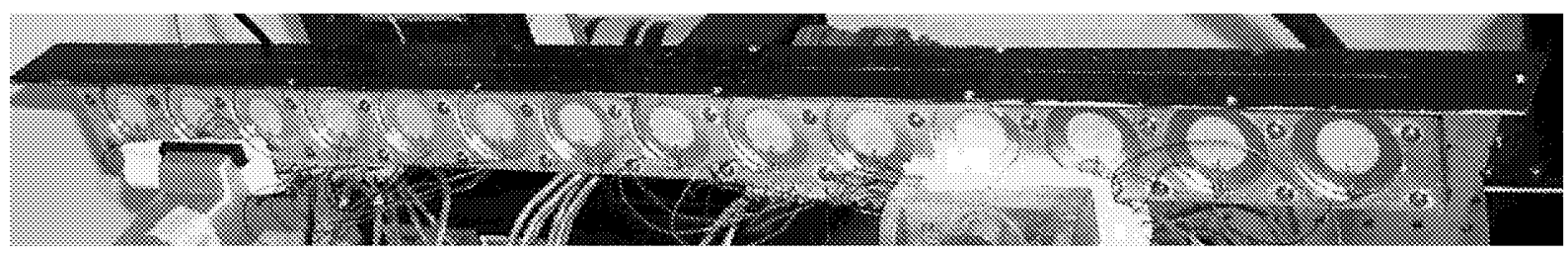

Figure 3. Photograph of the two-dimensional synthetic jet actuator on bench top. 


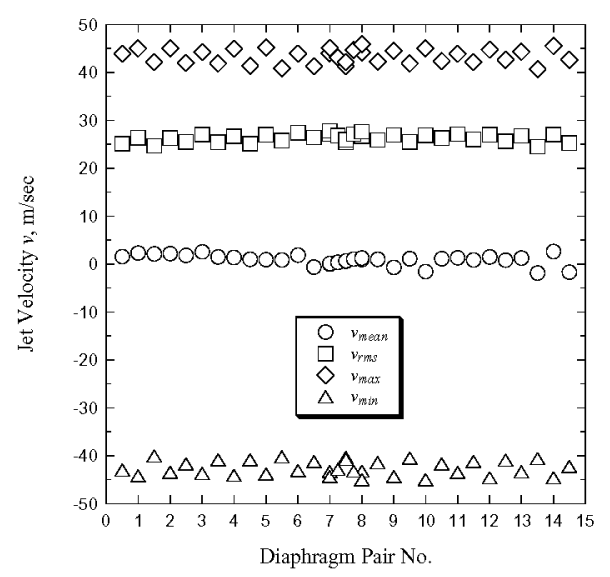

Figure 4. Variations of jet velocities along slot centerline on jet exit surface at $f=600 \mathrm{~Hz}$.

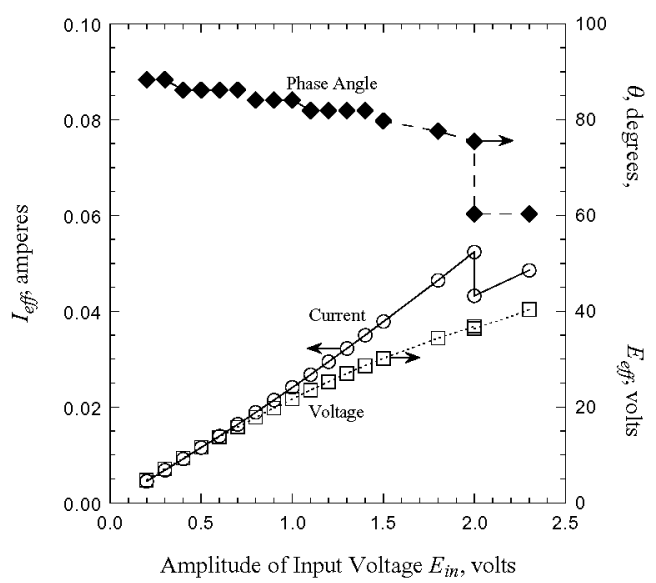

(a) Electric current, voltage and phase angle.

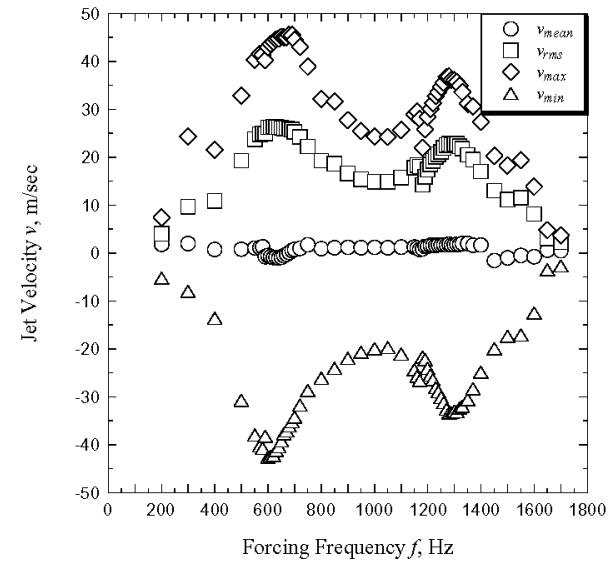

Figure 5. Variations of jet velocities with forcing frequency at slot center on jet exit surface.

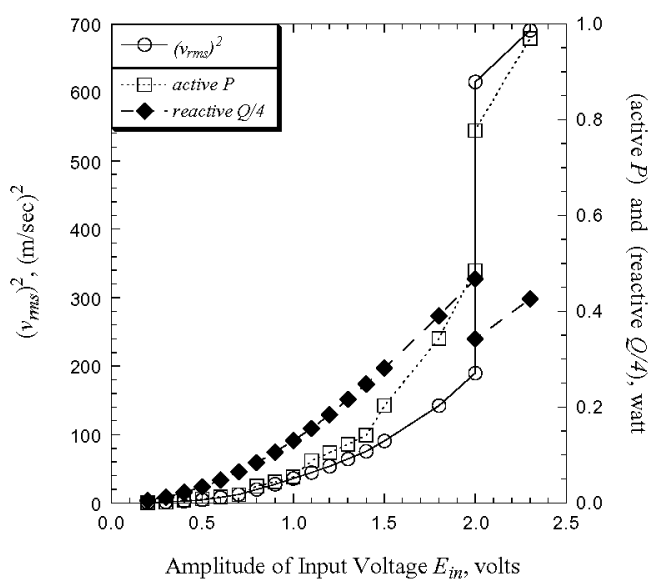

(b) Jet energy, active and reactive electric powers.

Figure 6. Characteristics of electric power consumption and jet energy output at forcing frequency $f=600 \mathrm{~Hz}$.

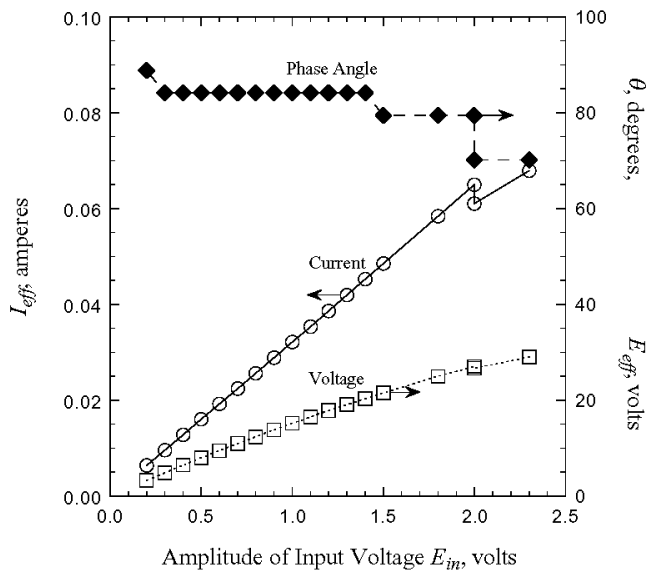

(a) Electric current, voltage and phase angle.

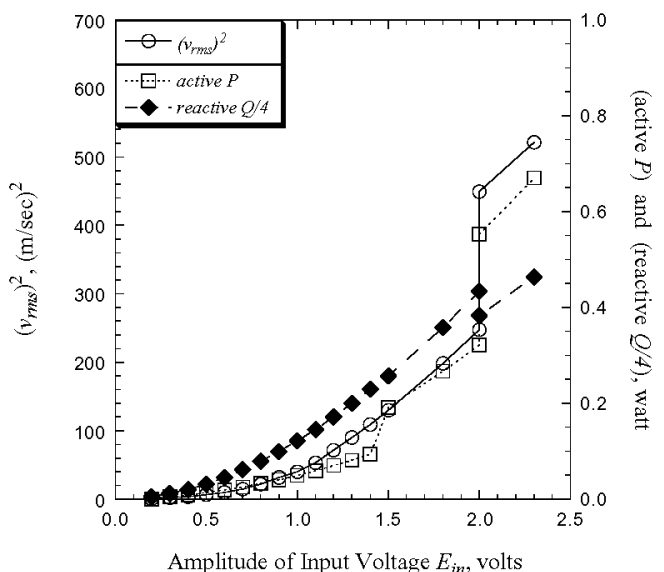

(b) Jet energy, active and reactive electric powers.

Figure 7. Characteristics of electric power consumption and jet energy output at forcing frequency $f=1300 \mathrm{~Hz}$. 


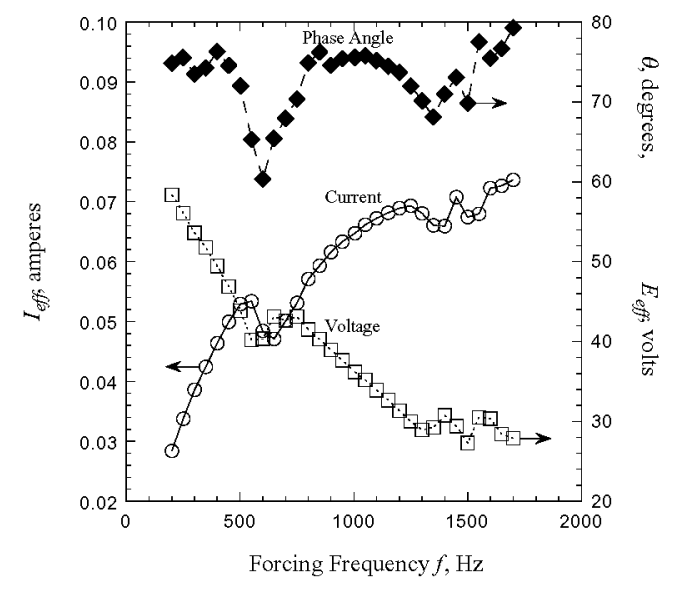

(a) Electric current, voltage and phase angle.

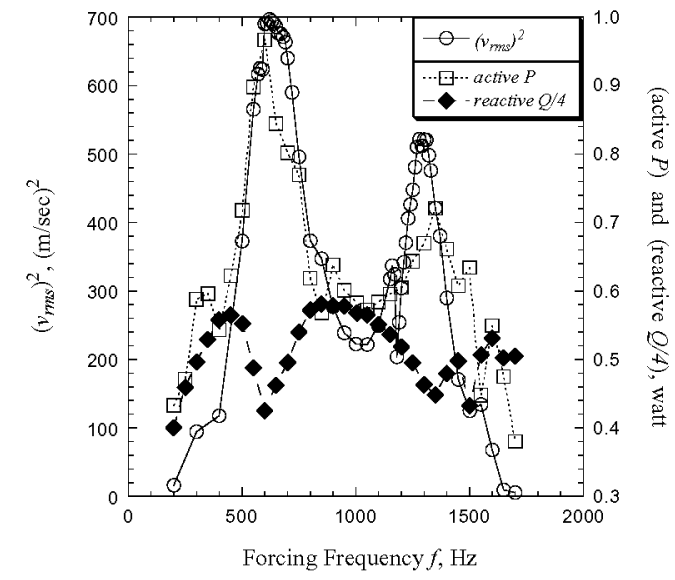

(b) Jet energy, active and reactive electric powers.

Figure 8. Variations of electric power consumption and jet energy output with synthetic jet forcing frequency.

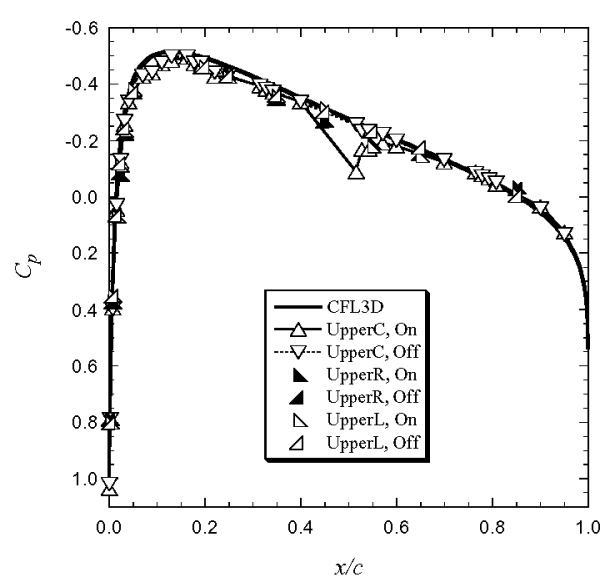

(a) $C_{p}$ on airfoil upper surface.

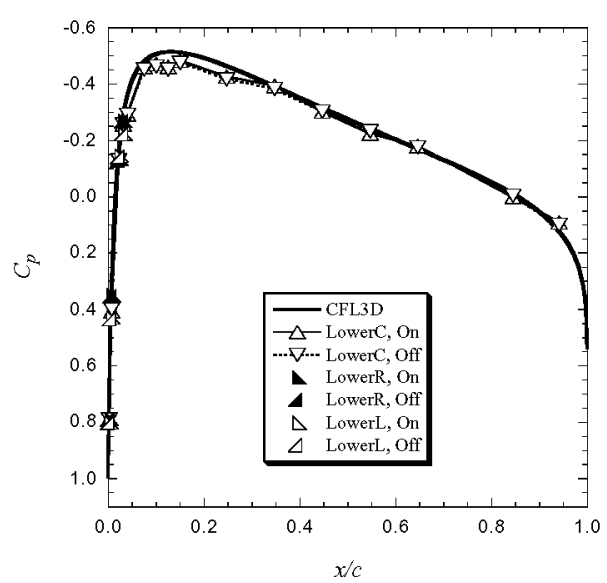

(b) $C_{p}$ on airfoil lower surface.

Figure 9. Variations of $C_{p}$ on airfoil surfaces with actuator located at $x / c=0.5$ on upper surface, operated at forcing frequency $f=600 \mathrm{~Hz}$ and freestream velocity $U_{\infty} \approx 10 \mathrm{~m} / \mathrm{sec}$.

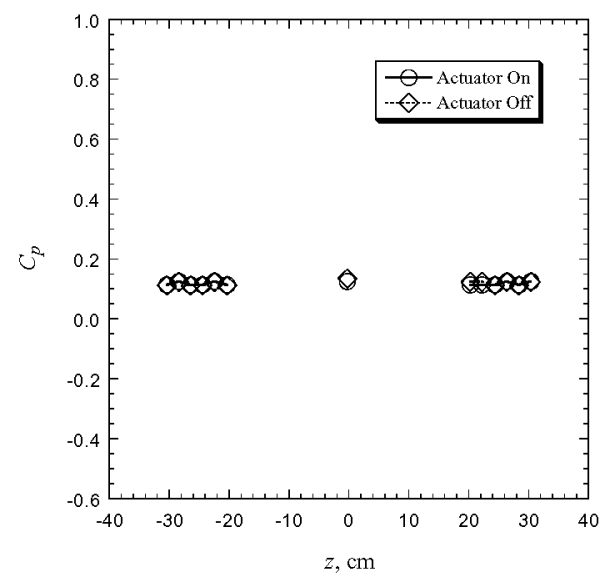

Figure 10. Variations of spanwise $C_{p}$ at $x / c=0.95$ on airfoil upper surface.

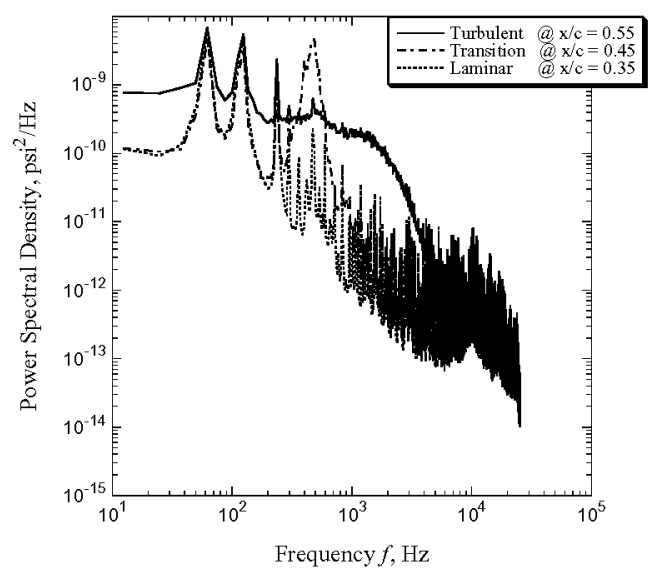

Figure 11. Power spectra of pressure fluctuations on airfoil upper surface for $U_{\infty} \approx 10 \mathrm{~m} / \mathrm{sec}$. 


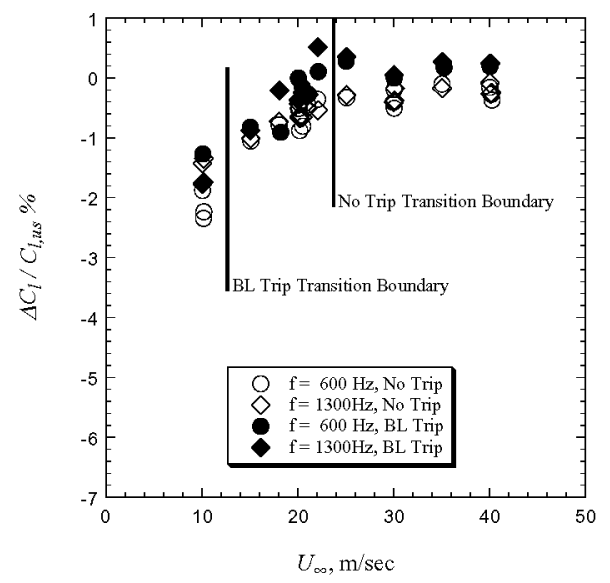

Figure 12. Effects of boundary-layer transition on virtual shaping when the actuator located at $x / c=0.15$ on upper surface.

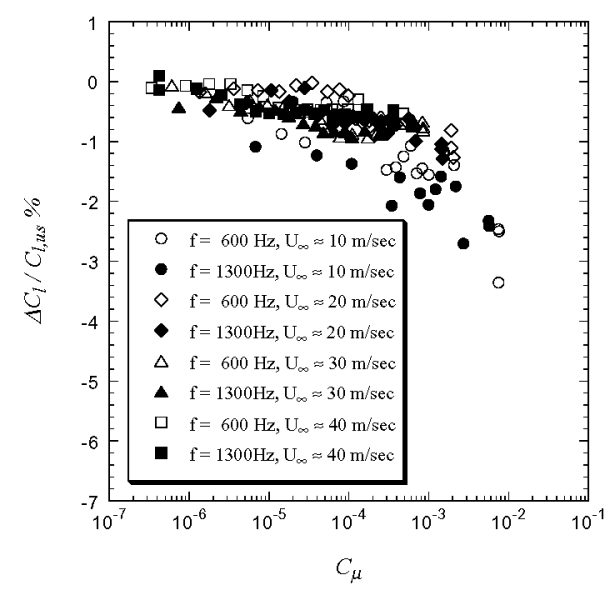

(a) Actuator at $x / c=0.1$.

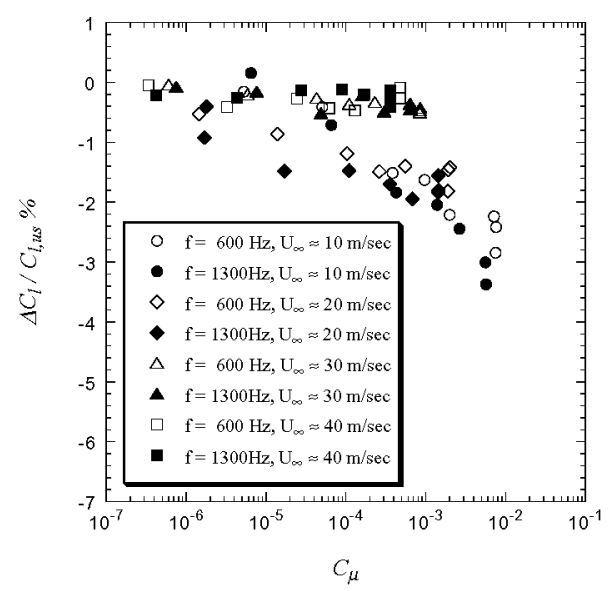

(c) Actuator at $x / c=0.3$.

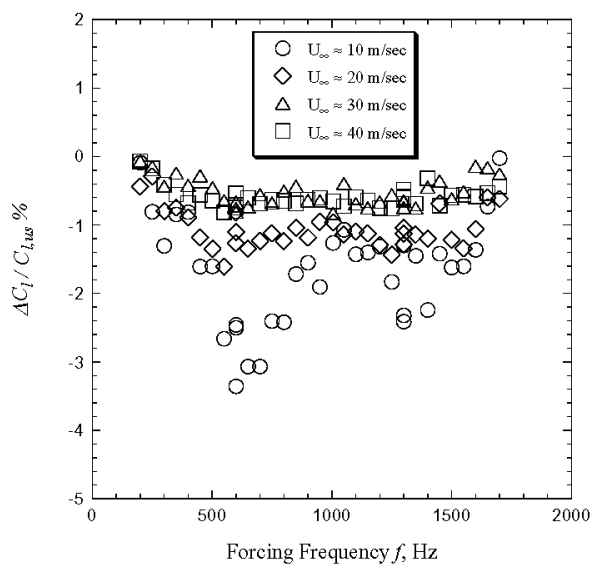

Figure 13. Variations of airfoil lift change with forcing frequency when the actuator located at $x / c=0.1$ on upper surface.

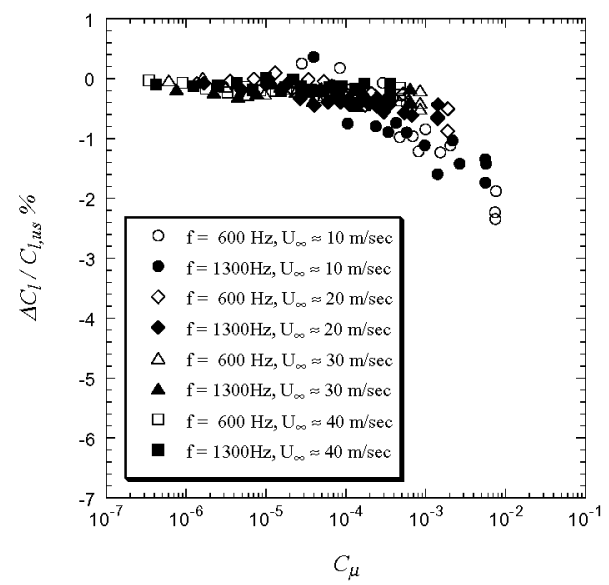

(b) Actuator at $x / c=0.15$.

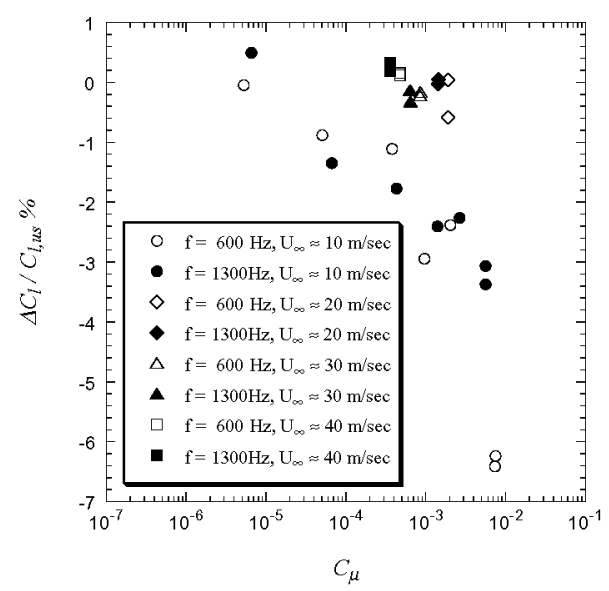

(d) Actuator at $x / c=0.5$.

Figure 14. Variations of airfoil lift change with jet momentum coefficient when the actuator located at different chordwise locations on the airfoil upper surface. 


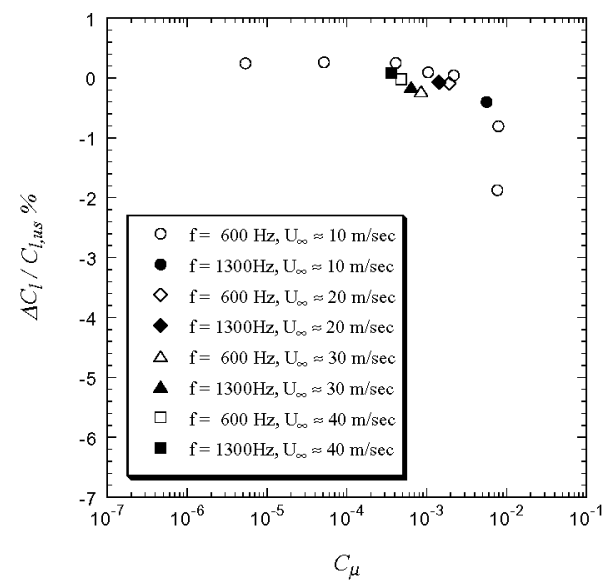

Figure 15. Variations of airfoil lift change with jet momentum coefficient when the actuator located at $x / c=0.75$ on upper surface.

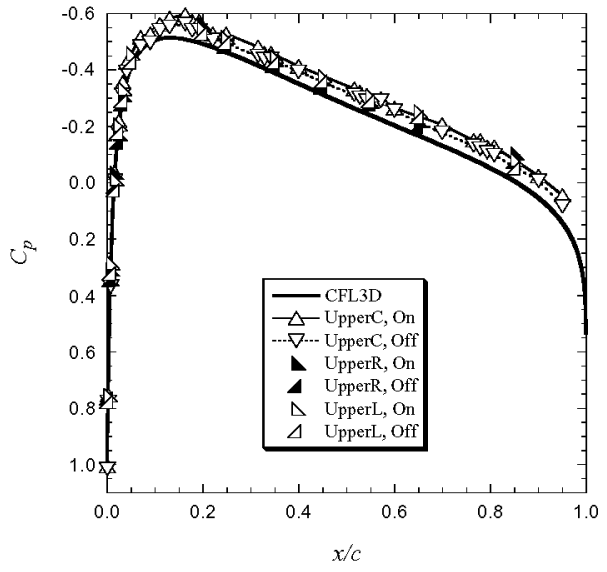

(a) $C_{p}$ on airfoil upper surface.

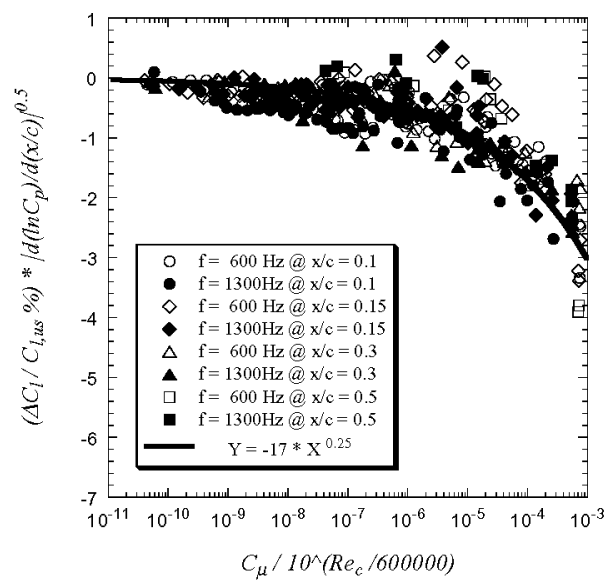

Figure 16. Correlation of airfoil lift change with jet momentum coefficient for data presented in Figures 14(a) (d).

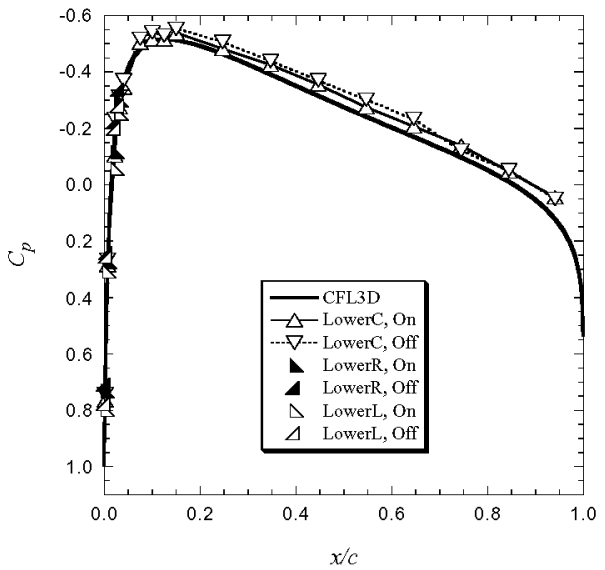

(b) $C_{p}$ on airfoil lower surface.

Figure 17. Variations of $C_{p}$ on airfoil surfaces with actuator located at $x / c=-0.015$ on upper surface, operated at forcing frequency $f=600 \mathrm{~Hz}$ and freestream velocity $U_{\infty} \approx 10 \mathrm{~m} / \mathrm{sec}$.

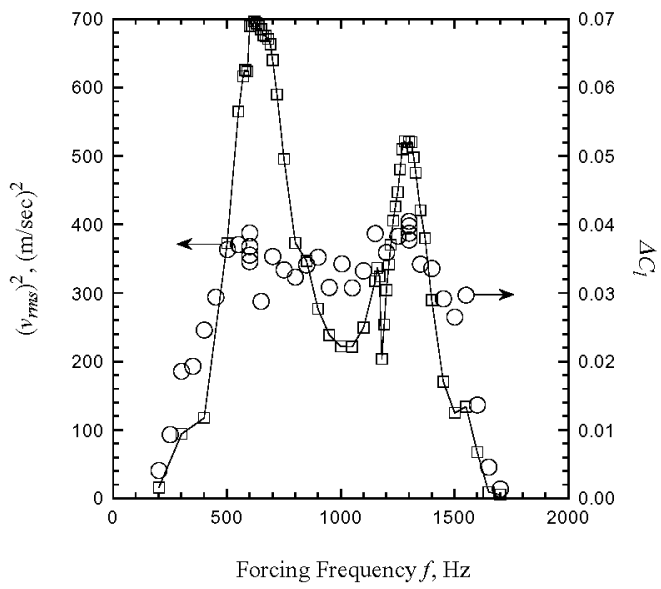

Figure 18. Variations of jet energy and airfoil lift change with forcing frequency when the actuator located at $x / c=-0.015$ and $U_{\infty} \approx 10 \mathrm{~m} / \mathrm{sec}$.

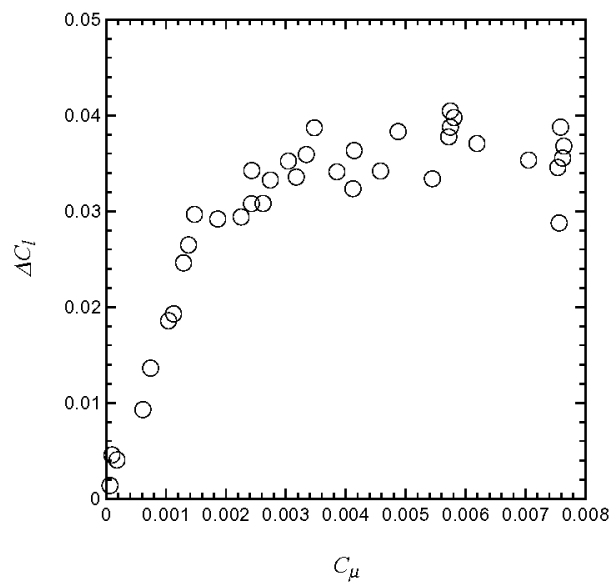

Figure 19. Variations of airfoil lift change with jet momentum coefficient when the actuator located at $x / c=-0.015$ and $U_{\infty} \approx 10 \mathrm{~m} / \mathrm{sec}$. 


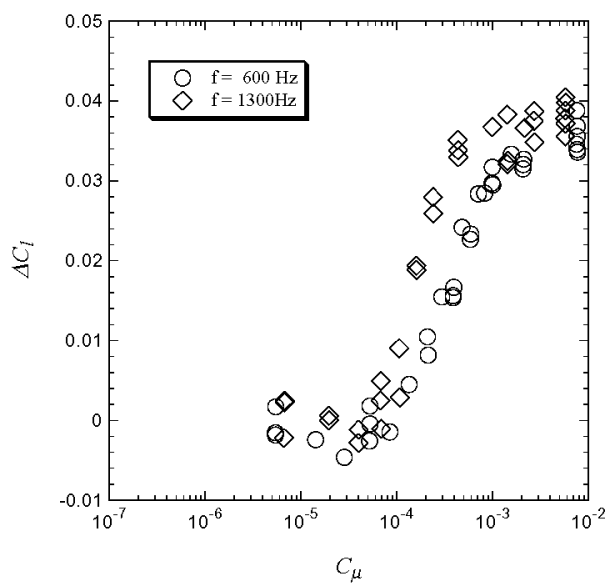

(a) $U_{\infty} \approx 10 \mathrm{~m} / \mathrm{sec}$.

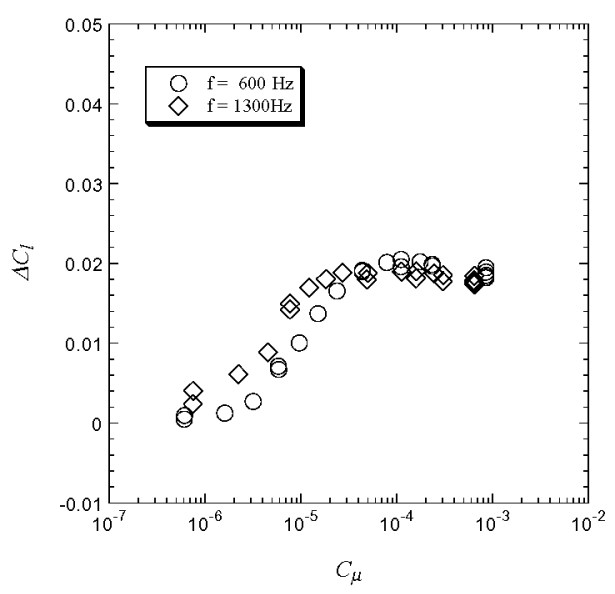

(c) $U_{\infty} \approx 30 \mathrm{~m} / \mathrm{sec}$.

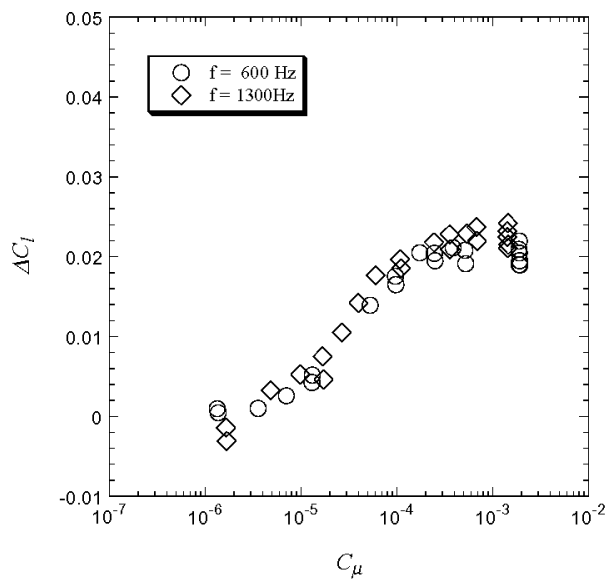

(b) $U_{\infty} \approx 20 \mathrm{~m} / \mathrm{sec}$.

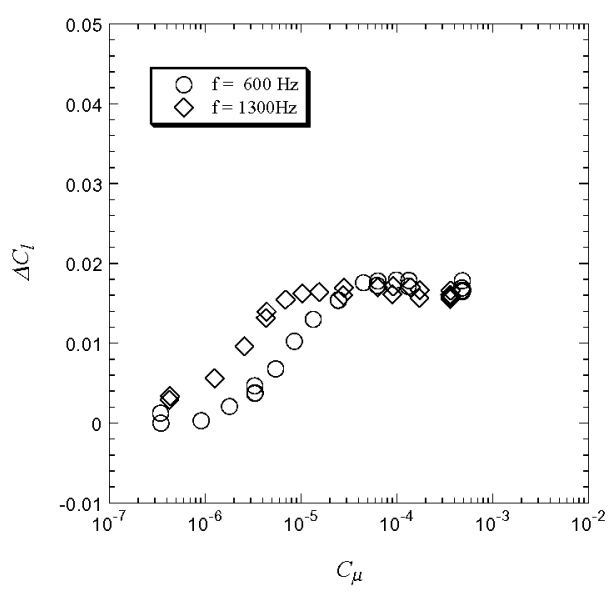

(d) $U_{\infty} \approx 40 \mathrm{~m} / \mathrm{sec}$.

Figure 20. Variations of airfoil lift change with jet momentum coefficient when the actuator located at $x / c=-0.015$ on airfoil lower surface.

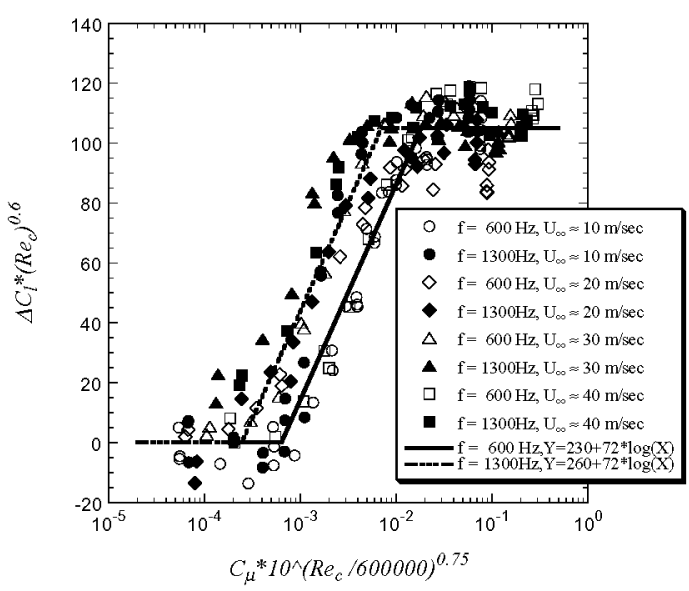

Figure 21. Correlation of airfoil lift change with jet momentum coefficient when the actuator located at $x / c=-0.015$ on airfoil lower surface. 